 \\ MOUNTaiNs \\ Mathematical Publications
}

\section{ON THE RATIONAL RECURSIVE SEQUENCE}

$$
x_{n+1}=\frac{a x_{n-1}}{b+c x_{n} x_{n-1}}
$$

\section{Anna Andruch-SobiŁo - Mągorzata Migda}

ABSTRACT. In this paper we consider the difference equation

$$
x_{n+1}=\frac{a x_{n-1}}{b+c x_{n} x_{n-1}}, \quad n=0,1, \ldots
$$

with positive parameters and nonnegative initial conditions. We use the explicit formula for the solutions of equation $(\mathrm{E})$ in investigating their behavior.

\section{Introduction}

In this paper we consider the following rational difference equation

$$
x_{n+1}=\frac{a x_{n-1}}{b+c x_{n} x_{n-1}}, \quad n=0,1, \ldots
$$

where $a, b, c$ are positive real numbers and the initial conditions $x_{-1}, x_{0}$ are nonnegative real numbers such that $x_{-1}$ or $x_{0}$ or both are positive real numbers. Equation $(\mathrm{E})$ in the case of negative $b$ was considered in [1].

The purpose of this paper is to use the explicit formula for solutions of equation $(\mathrm{E})$ in investigating their behavior. We will show that when $a<b$, the zero equilibrium is a global attractor for all positive solutions of equation (E) and that all positive solutions of equation (E) are bounded.

There has been a lot of work concerning the asymptotic behavior of solutions of rational difference equations. Second order rational difference equations were investigated, for example in [1-11]. This paper is motivated by the short notes [2] and [9], where the authors studied the rational difference equation

$$
x_{n+1}=\frac{x_{n-1}}{1+x_{n} x_{n-1}}, \quad n=0,1, \ldots
$$

2000 Mathematics Subject Classification: 39A10.

Keywords: rational difference equation, equilibrium point, explicit formula, boundedness, global asymptotic stability. 


\section{ANNA ANDRUCH-SOBILO - MAtGORZATA MIGDA}

\section{Main results}

Let $p=\frac{b}{a}, q=\frac{c}{a}$. Then equation (E) can be rewritten as

$$
x_{n+1}=\frac{x_{n-1}}{p+q x_{n} x_{n-1}}, \quad n=0,1, \ldots
$$

The change of variables $x_{n}=\frac{1}{\sqrt{q}} y_{n}$ reduces the above equation to

$$
y_{n+1}=\frac{y_{n-1}}{p+y_{n} y_{n-1}}, \quad n=0,1, \ldots
$$

where $p \in R_{+}$and the initial conditions $y_{-1}, y_{0}$ are nonnegative real numbers such that $y_{-1}$ or $y_{0}$ or both are positive real numbers. Hereafter, we focus our attention on equation (E2) instead of equation (E). Note, that the solution $\left\{y_{n}\right\}$ with $y_{-1}=0$ or $y_{0}=0$ of equation (E2) is oscillatory. In fact, in this case we have

or

$$
\left\{y_{n}\right\}=\left\{0, y_{0}, 0, \frac{y_{0}}{p}, 0, \frac{y_{0}}{p^{2}}, \ldots\right\}
$$

$$
\left\{y_{n}\right\}=\left\{y_{-1}, 0, \frac{y_{-1}}{p}, 0, \frac{y_{-1}}{p^{2}}, 0, \ldots\right\} .
$$

Obviously, if $p=1$, these solutions are 2-periodic.

Thus, let us assume that $y_{-1}$ and $y_{0}$ are positive. Then it is clear that $y_{n}>0$ for all $n \geq-1$. In the sequel, we will only consider positive solutions of equation (E2).

The equilibria of equation (E2) are the solutions of the equation

$$
\bar{y}=\frac{\bar{y}}{p+\bar{y}^{2}} .
$$

Hence, $\bar{y}=0$ is always an equilibrium point of equation (E2). Clearly, when $p \geq 1$ it is a unique equilibrium point. The local asymptotic behavior of the zero equilibrium of equation (E2) is characterized by the following result.

Theorem A ([11]). The following statements are true.

(i) If $p>1$, then $\bar{y}=0$ is locally asymptotically stable.

(ii) If $p<1$, then $\bar{y}=0$ is a repeller.

Applying Theorem 2.1 obtained by $\mathrm{C}$ in a r in [3] (with $a=b=\frac{1}{p}$ ) to equation (E2) we get the explicit formula for every solution $\left\{y_{n}\right\}$ with positive initial 
ON THE RATIONAL RECURSIVE SEQUENCE $x_{n+1}=\frac{a x_{n-1}}{b+c x_{n} x_{n-1}}$

conditions $y_{-1}, y_{0}$. We can write it in the following form

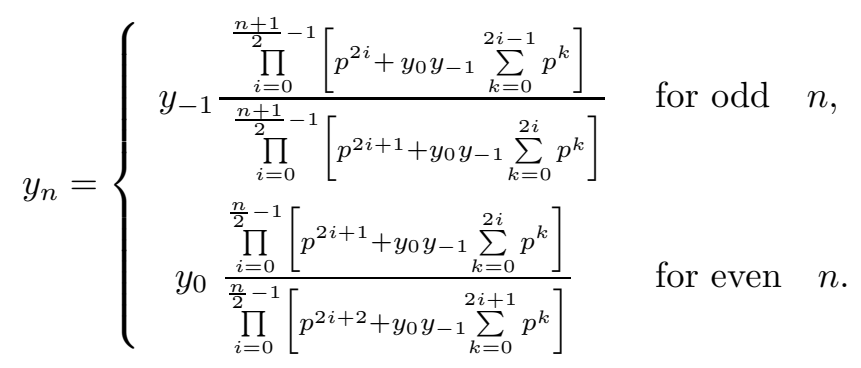

We will use the explicit formula for solutions of equation (E2) in investigating their asymptotic behavior. We will consider the cases, when $p \geq 1$ and $p \in(0,1)$.

Theorem 1. Assume that $p \geq 1$. Then every positive solution $\left\{y_{n}\right\}$ of equation (E2) converges to zero.

$\mathrm{Pr}$ o of. Let $\left\{y_{n}\right\}$ be a solution of equation (E2) satisfying the initial conditions $y_{-1}>0$ and $y_{0}>0$. It is enough to prove that the subsequences $\left\{y_{2 n}\right\}$ and $\left\{y_{2 n-1}\right\}$ converge to zero as $n \rightarrow \infty$. From (1) we have

$$
\begin{aligned}
y_{2 n}=y_{0} \frac{\prod_{i=0}^{n-1}\left[p^{2 i+1}+y_{0} y_{-1} \sum_{k=0}^{2 i} p^{k}\right]}{\prod_{i=0}^{n-1}\left[p^{2 i+2}+y_{0} y_{-1}^{2 i+1} p_{k=0}^{k}\right]} \\
=y_{0} \exp \left[\sum_{i=0}^{n-1} \ln \frac{p^{2 i+1}+y_{0} y_{-1} \sum_{k=0}^{2 i} p^{k}}{p^{2 i+2}+y_{0} y_{-1} \sum_{k=0}^{2 i+1} p^{k}}\right] \\
=y_{0} \exp \left[\sum_{i=0}^{n-1} \ln \left[1-\frac{p^{2 i+2}-p^{2 i+1}+p^{2 i+1} y_{0} y_{-1}}{p^{2 i+2}+y_{0} y_{-1} \sum_{k=0}^{2 i+1} p^{k}}\right)\right] \\
\leq y_{0} \exp \left[-\sum_{i=0}^{n-1} \frac{p^{2 i+2}-p^{2 i+1}+p^{2 i+1} y_{0} y_{-1}}{p^{2 i+2}+y_{0} y_{-1}^{2 i+1} \sum_{k=0}^{k}}\right] \\
=y_{0} \exp \left[-\sum_{i=0}^{n-1} \frac{p^{2 i+1}\left(p-1+y_{0} y_{-1}\right)}{p^{2 i+2}+y_{0} y_{-1} \sum_{k=0}^{2 i+1} p^{k}}\right] .
\end{aligned}
$$




\section{ANNA ANDRUCH-SOBILO - MAtGORZATA MIGDA}

So, we have

$$
y_{2 n} \leq y_{0} \exp \left[-\left(p-1+y_{0} y_{-1}\right) \sum_{i=0}^{n-1} \frac{p^{2 i+1}}{p^{2 i+2}+y_{0} y_{-1} \sum_{k=0}^{2 i+1} p^{k}}\right] .
$$

Since $p \geq 1, p-1+y_{0} y_{-1}>0$ and from the above inequality we obtain

$$
\begin{aligned}
y_{2 n} & \leq y_{0} \exp \left[-\left(p-1+y_{0} y_{-1}\right) \sum_{i=0}^{n-1} \frac{p^{2 i+1}}{p^{2 i+2}+y_{0} y_{-1} p^{2 i+1} \sum_{k=0}^{2 i+1} 1}\right] \\
& =y_{0} \exp \left[-\left(p-1+y_{0} y_{-1}\right) \sum_{i=0}^{n-1} \frac{1}{p+y_{0} y_{-1}(2 i+2)}\right] .
\end{aligned}
$$

Because $\sum_{i=0}^{n-1} \frac{1}{p+y_{0} y_{-1}(2 i+2)} \rightarrow \infty$ as $n \rightarrow \infty$, so $y_{2 n} \rightarrow 0$ as $n \rightarrow \infty$.

Similarly, we obtain

$y_{2 n-1} \leq y_{-1} \exp \left[-\left(p-1+y_{0} y_{-1}\right) \sum_{i=0}^{n-1} \frac{1}{p+y_{0} y_{-1}(2 i+1)}\right] \rightarrow 0, \quad$ as $\quad n \rightarrow \infty$.

This completes the proof.

Theorem 1 extends Theorem 1 of Stević [9].

Theorem 1 and Theorem A imply the following result.

Corollary 1. Assume $p>1$. Then the unique equilibrium $\bar{y}=0$ of equation (E2) is globally asymptotically stable.

Note, that equation (E2) is a special case of equation (3.3) in [11], but our result relating the global attractivity of the zero equilibrium is stronger.

For $p \in(0,1)$ we have the following result about the subsequences of the even terms $\left\{y_{2 n}\right\}_{n=0}^{\infty}$ and the odd terms $\left\{y_{n}\right\}_{n=-1}^{\infty}$ of every positive solution $\left\{y_{n}\right\}$ of equation (E2).

TheOREM 2. Assume that $p \in(0,1)$. Let $\left\{y_{n}\right\}$ be a solution of equation (E2) with positive initial conditions $y_{-1}, y_{0}$. Then the following statements are true:

(i) If $y_{0} y_{-1}<1-p$, then the subsequences $\left\{y_{2 n}\right\}$ and $\left\{y_{2 n-1}\right\}$ are both increasing and bounded.

(ii) If $y_{0} y_{-1}>1-p$, then the subsequences $\left\{y_{2 n}\right\}$ and $\left\{y_{2 n-1}\right\}$ are both decreasing and bounded.

(iii) If $y_{0} y_{-1}=1-p$, then the subsequences $\left\{y_{2 n}\right\}$ and $\left\{y_{2 n-1}\right\}$ are both constant sequences. 
ON THE RATIONAL RECURSIVE SEQUENCE $x_{n+1}=\frac{a x_{n-1}}{b+c x_{n} x_{n-1}}$

Proof.

(i) Let $\left\{y_{n}\right\}$ be a positive solution of equation (E2). From (11) for the subsequence $\left\{y_{2 n}\right\}$ we have

$$
y_{2 n}=y_{0} \frac{\prod_{i=0}^{n-1}\left[p^{2 i+1}+y_{0} y_{-1} \sum_{k=0}^{2 i} p^{k}\right]}{\prod_{i=0}^{n-1}\left[p^{2 i+2}+y_{0} y_{-1} \sum_{k=0}^{2 i+1} p^{k}\right]},
$$

and so for $n \geq 0$

$$
\begin{aligned}
\frac{y_{2 n+2}}{y_{2 n}}= & \frac{\prod_{i=0}^{n}\left[p^{2 i+1}+y_{0} y_{-1} \sum_{k=0}^{2 i} p^{k}\right] \prod_{i=0}^{n-1}\left[p^{2 i+2}+y_{0} y_{-1} \sum_{k=0}^{2 i+1} p^{k}\right]}{\prod_{i=0}^{n}\left[p^{2 i+2}+y_{0} y_{-1} \sum_{k=0}^{2 i+1} p^{k}\right] \prod_{i=0}^{n-1}\left[p^{2 i+1}+y_{0} y_{-1} \sum_{k=0}^{2 i} p^{k}\right]} \\
= & \frac{p^{2 n+1}+y_{0} y_{-1} \sum_{k=0}^{2 n} p^{k}}{p^{2 n+2}+y_{0} y-1 \sum_{k=0}^{2 n+1} p^{k}}
\end{aligned}
$$

Since $y_{0} y_{-1}<1-p$, we have

$$
y_{0} y_{-1} p^{2 n+1}<p^{2 n+1}-p^{2 n+2} .
$$

Hence

$$
y_{0} y_{-1}\left(\sum_{k=0}^{2 n+1} p^{k}-\sum_{k=0}^{2 n} p^{k}\right)<p^{2 n+1}-p^{2 n+2},
$$

and therefore

$$
p^{2 n+1}+y_{0} y_{-1} \sum_{k=0}^{2 n} p^{k}>p^{2 n+2}+y_{0} y_{-1} \sum_{k=0}^{2 n+1} p^{k} .
$$

From the above inequality and (3) it follows that the subsequence $\left\{y_{2 n}\right\}$ is increasing. Similarly we obtain that the subsequence $\left\{y_{2 n-1}\right\}$ is increasing. Now, we will show that the solution $\left\{y_{n}\right\}$ is bounded.

From (2) we have

$$
\begin{aligned}
y_{2 n} & \leq y_{0} \exp \left[-\left(p-1+y_{0} y_{-1}\right) \sum_{i=0}^{n-1} \frac{p^{2 i+1}}{p^{2 i+2}+y_{0} y_{-1} \sum_{k=0}^{2 i+1} p^{k}}\right] \\
& =y_{0} \exp \left[\left(1-p-y_{0} y_{-1}\right)(1-p) \sum_{i=0}^{n-1} \frac{p^{2 i+1}}{p^{2 i+2}(1-p)+y_{0} y_{-1}\left(1-p^{2 i+2}\right)}\right] .
\end{aligned}
$$


Since for $p \in(0,1)$ the series

$$
\sum_{i=0}^{n-1} \frac{p^{2 i+1}}{p^{2 i+2}(1-p)+y_{0} y_{-1}\left(1-p^{2 i+2}\right)}
$$

is convergent and we get the boundedness of $\left\{y_{2 n}\right\}$. Similarly we obtain the boundedness of the subsequence $\left\{y_{2 n-1}\right\}$.

(ii) The proof is similar to the proof of $(i)$ and will be omitted.

(iii) If $y_{0} y_{-1}=1-p$ then from (E2) we get

$$
y_{n+1}=\frac{y_{n-1}}{p+y_{n} y_{n-1}}=y_{n-1} \text {. }
$$

Hence

$$
\left\{y_{2 n}\right\}=\left\{y_{0}, y_{0}, y_{0}, \ldots\right\} \quad \text { and } \quad\left\{y_{2 n-1}\right\}=\left\{y_{-1}, y_{-1}, y_{-1}, \ldots\right\}
$$

and, the solution

$$
\left\{y_{n}\right\}=\left\{y_{-1}, y_{0}, y_{-1}, y_{0}, \ldots y_{-1}, y_{0}\right\}
$$

is 2-periodic.

This completes the proof.

From Theorem 1 and Theorem 2 we get the following corollary.

Corollary 2. Every positive solution of equation (E) is bounded.

We say the sequences $\left\{a_{n}\right\},\left\{b_{n}\right\}$ are equivalent (Cauchy equivalent) if $\lim _{n \rightarrow \infty}\left(a_{n}-b_{n}\right)=0$. If there exists a $k$-periodic sequence $\left\{c_{n}\right\}$ equivalent to $\left\{a_{n}\right\}$, we say that $\left\{a_{n}\right\}$ is asymptotically $k$-periodic sequence.

The next corollary follows from Theorem 2 and from the expression of equation (E2).

Corollary 3. Assume that $p \in(0,1)$. Then every positive solution of equation (E2) is asymptotically 2-periodic sequence $\{r, s, r, s, r, s, \ldots\}$, where $r s=$ $1-p$.

Moreover, it is clear from formula (11) that, for a fixed $p$, numbers $r$ and $s$ depend only on the initial data $y_{-1}, y_{0}$.

\section{Numerical results}

ExAMPLE 1. Let $y_{-1}=2, y_{0}=5$ be the initial conditions of equation (E2) with $p=2$. Then, by Theorem 1, the solution converges to zero.

The Table 1 sets forth the values of $y_{n}$ for selected small $n$ 's. Note that Theorem 5.2 from [11] in this case can not be applied. 
ON THE RATIONAL RECURSIVE SEQUENCE $x_{n+1}=\frac{a x_{n-1}}{b+c x_{n} x_{n-1}}$

TABLE 1 . The values of $y_{n}$ for selected small $n$ 's.

\begin{tabular}{|c|c|c|c|}
\hline $\mathrm{n}$ & $y_{n}$ & $\mathrm{n}$ & $y_{n}$ \\
\hline 1 & 0.1666666666 & 2 & 1.764705882 \\
\hline 3 & 0.07264957264 & 4 & 0.8291991495 \\
\hline 5 & 0.03526265806 & 6 & 0.4086255174 \\
\hline 7 & 0.01750521080 & 8 & 0.2035846305 \\
\hline 9 & 0.008737036910 & 10 & 0.1017018654 \\
\hline 29 & $8.527211545 * 10^{-6}$ & 30 & $9.928883343 * 10^{-5}$ \\
\hline 699 & $1.218311909 * 10^{-106}$ & 700 & $1.418573558 * 10^{-105}$ \\
\hline
\end{tabular}

EXAMPLE 2. Let $y_{-1}=500, y_{0}=100$ be the initial conditions of equation (E2) with $p=\frac{4}{5}$. Then $y_{0} y_{-1}=50000,1-p=\frac{1}{5}$. So condition $y_{0} y_{-1}>1-p$ holds and by Theorem 2 , the subsequences $\left\{y_{2 n}\right\}$ and $\left\{y_{2 n-1}\right\}$ are both decreasing.

The Table 2 sets forth the values of $y_{n}$ for selected small $n$ 's.

TABLE 2. The values of $y_{n}$ for selected small $n$ 's.

\begin{tabular}{|c|c|c|c|}
\hline $\mathrm{n}$ & $y_{n}$ & $\mathrm{n}$ & $y_{n}$ \\
\hline 1 & 0.009999840002 & 2 & 55.55604937 \\
\hline 3 & 0.007376952648 & 4 & 45.92037709 \\
\hline 5 & 0.006478100367 & 6 & 41.84177433 \\
\hline 7 & 0.006048334655 & 8 & 39.73302154 \\
\hline 9 & 0.005813925263 & 10 & 38.53815313 \\
\hline 29 & 0.005460160780 & 30 & 36.67435849 \\
\hline 49 & 0.005456447604 & 50 & 36.65440759 \\
\hline 99 & 0.005456404175 & 100 & 36.65417618 \\
\hline
\end{tabular}

EXAMPLE 3. Let $y_{-1}=0.001, y_{0}=2$ be the initial conditions of equation (E2) with $p=0.9$. Then $y_{0} y_{-1}=0.002,1-p=0.1$. So condition $y_{0} y_{-1}<1-p$ holds and by Theorem 2 , the subsequences $\left\{y_{2 n}\right\}$ and $\left\{y_{2 n-1}\right\}$ are both increasing.

The Table 3 sets forth the values of $y_{n}$ for selected small $n$ 's. 


\section{ANNA ANDRUCH-SOBILO - MAtGORZATA MIGDA}

TABLE 3. The values of $y_{n}$ for selected small $n$ 's.

\begin{tabular}{|c|c|c|c|}
\hline $\mathrm{n}$ & $y_{n}$ & $\mathrm{n}$ & $y_{n}$ \\
\hline 1 & 0.00110864745 & 2 & 2.216760874 \\
\hline 3 & 0.001228475933 & 4 & 2.455637323 \\
\hline 5 & 0.001360413317 & 6 & 2.718395588 \\
\hline 7 & 0.001505384658 & 8 & 3.006767998 \\
\hline 9 & 0.00166427951 & 10 & 3.322380518 \\
\hline 25 & 0.003484539186 & 26 & 6.888785559 \\
\hline 49 & 0.006449046007 & 50 & 12.37982939 \\
\hline 99 & 0.007251009859 & 100 & 13.77325644 \\
\hline 299 & 0.007255975296 & 300 & 13.78174482 \\
\hline
\end{tabular}

Acknowledgements. The authors thank the referee for valuable comments.

\section{REFERENCES}

[1] ANDRUCH-SOBIŁO, A.-MIGDA, M.: Further properties of the rational recursive sequence $x_{n+1}=\frac{a x_{n-1}}{b+c x_{n} x_{n-1}}$, Opuscula Math. 26 (2006), 387-394.

[2] CINAR, C.: On the positive solutions of the difference equation $x_{n+1}=\frac{x_{n-1}}{1+x_{n} x_{n-1}}$, Appl. Math. Comput. 150 (2004), 21-24.

[3] CINAR, C.: On the positive solutions of the difference equation $x_{n+1}=\frac{a x_{n-1}}{1+b x_{n} x_{n-1}}$, Appl. Math. Comput. 156 (2004), 587-590.

[4] CHANG, D. C.-STEVIĆ, S.: On the recursive sequence $x_{n+1}=\alpha+\frac{\beta x_{n-1}}{1+g\left(x_{n}\right)}$, Appl. Anal. 82 (2003), 145-156.

[5] EL-OWAIDY, H. M.-AHMED, A. M.-MOOUSA, M. S.: On the recursive sequences $x_{n+1}=\frac{-\alpha x_{n-1}}{\beta \pm x_{n}}$, Appl. Math. Comput. 145 (2003), 747-753.

[6] KOSMALA, W. A.-KUlEnOVIC, M. R. S.-LADAS, G.-TEIXEIRA, C. T.: On the recursive sequence $y_{n+1}=\frac{p+y_{n-1}}{q y_{n}+y_{n-1}}$, J. Math. Anal. Appl. 251 (2000), 571-586.

[7] LI, W. T.-SUN, H. R.: Dynamics of rational difference equation, Appl. Math. Comput. 163 (2005), 577-591.

[8] HUANG, Y. S.-KNOPF, P. M.: Bondedness of positive solutions of second-order rational difference equations, J. Difference Equ. Appl. 10 (2004), 935-940.

[9] STEVIĆ, S.: More on a rational recurrence relation, Appl. Math. E-Notes 4 (2004), 80-84.

[10] YAN, X.-LI, W.: Global attractivity in the recursive sequence $x_{n+1}=\frac{\alpha-\beta x_{n}}{\gamma-x_{n-1}}$, Appl. Math. Comput. 138 (2003), 415-423. 
ON THE RATIONAL RECURSIVE SEQUENCE $x_{n+1}=\frac{a x_{n-1}}{b+c x_{n} x_{n-1}}$

[11] YANG, X.-SU, W.-CHEN, B.-MEGSON, G.-EVANS, D.: On the recursive sequences $x_{n}=\frac{a x_{n-1}+b x_{n-2}}{c+d x_{n-1} x_{n-2}}$, Appl. Math. Comput. 162 (2005), 1485-1497.

Received August 19, 2008

\author{
Institute of Mathematics \\ Poznań University of Technology \\ Piotrowo $3 \mathrm{~A}$ \\ PL-60-965 Poznań \\ POLAND
}

E-mail: anna.andruch-sobilo@put.poznan.pl malgorzata.migda@put.poznan.pl 